\title{
Optimized Waveform Relaxation Solution of Electromagnetic and Circuit Problems
}

\author{
Martin J. Gander* and Albert E. Ruehli ${ }^{+}$, \\ ${ }^{*}$ Section of Mathematics, University of Geneva, Switzerland \\ ${ }^{+}$EMC Lab Missouri Univ. Science and Tech., Rolla, MO \\ Emeritus, IBM Research, Yorktown Heights, NY \\ albert.ruehli@gmail.com
}

\begin{abstract}
New algorithms are needed to solve electromagnetic problems using today's widely available parallel processors. In this paper, we show that applying the optimized waveform relaxation approach to a partial element equivalent circuit will yield a powerful technique for solving electromagnetic problems with the potential for a large number of parallel processor nodes.
\end{abstract}

\section{INTRODUCTION}

In this paper, we introduce a method for the parallel solution of time domain combined ElectroMagetic (EM) and circuit problems. The EM part is represented with a Partial Element Equivalent Circuit (PEEC) model [1]. This transforms the EM part into an equivalent circuit model. The PEEC model is solved with the Modified Nodal Analysis (MNA) technique, that is also used in most Spice circuit solvers. Hence, this approach also leads to an EM solution which includes $d c$ solutions important for chip and package applications.

The classical Waveform Relaxation (WR) approach was conceived in 1981 for circuit solver applications [2]. The approach is based on partitioning large circuits into many small circuits which then are solved separately on small Spice solvers. Sophisticated partitioning algorithms must be used with the classical WR as has been pointed out in [3]. The classical WR approach has a rich history of different techniques applied to a multitude of problems summarized in [4]. In this paper, we use a new form of WR called optimized WR (oWR) using optimized transmission conditions between the subsystems. Basically, oWR is a subclass of domain decomposition techniques. The oWR approach was conceived for PDEs in [5], see also [6], [7]. It has also been applied to the circuit domain e.g. [8], [9]. For a related approach, see also [10], [11].

Fundamentally, the approach divides the system matrix into as many subsystems (SSy) as are needed for the problem at hand. This permits the use of a large number of parallel processors. Further, the approach communicates waveforms in time between processors rather than transmitting the information at time points as is the case with other techniques. Also, the compute time for each SSy solution is not minute as is the case for some other algorithms, since we compute multiple time points or waveforms in time. Hence, the processor to processor communication latency is not as important for WR as it is

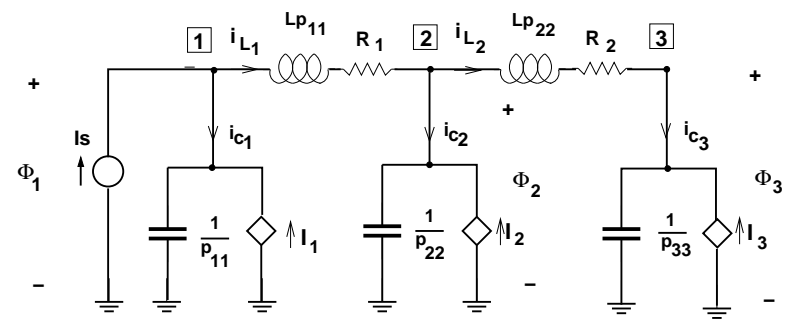

Fig. 1. Basic two stages PEEC circuit model

for other algorithms. These aspects are of importance, for the efficient use of a large number of processors. Such algorithms are not easy to find as has been pointed out in [12]. Recent work for EM and circuit related works using the classical WR approach has yielded good results. The WR algorithm was applied to several electromagnetic problems, e.g., [13] where it was shown that the classical WR leads to a very efficient solution for the transverse partitioning of multiple transmission lines. As another example, the approach was applied to antenna arrays with a large number of elements [14].

The optimized WR approach is based on an improved transmission condition between SSys. In the classical WR, different approaches have been experimented with for the information exchange between SSys. Techniques, some of which are related to overlap partitioning for circuits, are referenced in the overview paper [4]. These approaches are related to overlapping domains in domain decomposition methods, see [7]. The new transmission conditions use a combination of multiple variables with optimized constants or variables where the convergence is sped up. This results in considerable improvement in convergence. Even more important, we have shown that circuits which did not converge with classical WR can be made to converge relatively fast with oWR. An important issue is the choice of the optimization parameters. This issue is much more manageable for the known topology of a PEEC circuit. The studies in earlier work have helped us to better understand the choice of the parameters. Fortunately, the fast convergence issue is not too sensitive to the choice of the optimization parameters. In earlier work we viewed the parameters in terms of equations. In this paper, we also have a circuit interpretation which considerably helps the understanding of the concepts. 


\section{SMALL MODEL CIRCUIT}

We consider a few PEEC sections since general circuits consist of a multitude of them. For the analytical case, it is sufficient to model the circuit for two PEEC sections shown in Fig. 1. We set up the circuit equations to determine the best oWR model. The details of this model are available in [1]. The delay equations for the capacitance are given by

$$
\begin{aligned}
I_{1} & =\frac{p_{12}}{p_{11}} i c_{2}\left(t-\tau_{12}\right)+\frac{p_{13}}{p_{11}} i c_{3}\left(t-\tau_{13}\right), \\
I_{2} & =\frac{p_{21}}{p_{22}} i c_{1}\left(t-\tau_{12}\right)+\frac{p_{23}}{p_{22}} i c_{3}\left(t-\tau_{23}\right), \\
I_{3} & =\frac{p_{31}}{p_{33}} i c_{1}\left(t-\tau_{13}\right)+\frac{p_{32}}{p_{33}} i c_{2}\left(t-\tau_{23}\right) .
\end{aligned}
$$

At node 1 , we get the KCL equations

$$
i_{c 1}=-i_{L 1}+I_{s},
$$

and for the intermediate node 2 , solved for the capacitance current

$$
i_{c 2}=i_{L 1}-i_{L 2}
$$

Finally, the capacitive current for node 3 is simply

$$
i_{c 3}=i_{L 2} \text {. }
$$

Inserting (2) and (3) into (1), we obtain

$$
\begin{aligned}
I_{1} & =\frac{p_{12}}{p_{11}}\left[I_{L}\left(t-\tau_{L 1}\right)-I_{L 2}\left(t-\tau_{L}\right)\right]+\frac{p_{13}}{p_{11}} i_{L 2}\left(t-\tau_{L}\right), \\
I_{2} & =\frac{p_{21}}{p_{22}} i c_{1}\left(t-\tau_{12}\right)+\frac{p_{23}}{p_{22}} i c_{3}\left(t-\tau_{23}\right), \\
I_{3} & =\frac{p_{31}}{p_{33}} i c_{1}\left(t-\tau_{13}\right)+\frac{p_{32}}{p_{33}} i c_{2}\left(t-\tau_{23}\right) .
\end{aligned}
$$

For the admittance part of the MNA equations, we have

$$
\begin{aligned}
-I_{s}+\frac{1}{p_{11}} \frac{d \Phi_{1}}{d t}-I_{1}+i_{L 1} & =0, \\
-i_{L 1}+\frac{1}{p_{22}} \frac{d \Phi_{2}}{d t}-I_{2}+i_{L 2} & =0 \\
-i_{L 2}+\frac{1}{p_{33}} \frac{d \Phi_{3}}{d t}-I_{3} & =0 .
\end{aligned}
$$

The frequency domain MNA circuit matrix $M$ for this case is therefore given by

$$
\left[\begin{array}{ccccc}
\frac{s}{p_{11}} & 1-\frac{p_{12}}{p_{11}} e^{-s \tau_{12}} & 0 & \frac{p_{12}}{p_{11}} e^{-s \tau_{12}}-\frac{p_{13}}{p_{11}} e^{-s \tau_{13}} & 0 \\
1 & -s L p_{11}-R_{1} & -1 & -s L p_{12} e^{-s \tau_{L}} & 0 \\
0 & 1-\frac{p_{21}}{p_{22}} e^{-s \tau_{12}} & \frac{s}{p_{22}} & 1-\frac{p_{23}}{p_{22}} e^{-s \tau_{23}} & 0 \\
0 & -s L p_{21} e^{-s \tau_{L}} & 1 & -s L p_{22}-R_{2} & -1 \\
0 & \frac{p_{31}}{p_{33}} e^{-s \tau_{13}}-\frac{p_{32}}{p_{33}} e^{-s \tau_{23}} & 0 & -1+\frac{p_{32}}{p_{33}} e^{-s \tau_{23}} & \frac{s}{p_{33}}
\end{array}\right]
$$

and the corresponding system is

$$
M \boldsymbol{x}=\boldsymbol{b},
$$

with

$$
\boldsymbol{x}=\left[\begin{array}{lllll}
\Phi_{1} & i_{L 1} & \Phi_{2} & i_{L 2} & \Phi_{3}
\end{array}\right]^{T}
$$

and

$$
\boldsymbol{b}=\left[\begin{array}{lllll}
I_{s} & 0 & \frac{p_{21}}{p_{22}} I_{s} e^{-s \tau_{21}} & 0 & \frac{p_{31}}{p_{33}} e^{-s \tau_{13}}
\end{array}\right]^{T} .
$$

Hence, the solution for the model in Fig. 1 in the frequency domain is defined by the system (7).

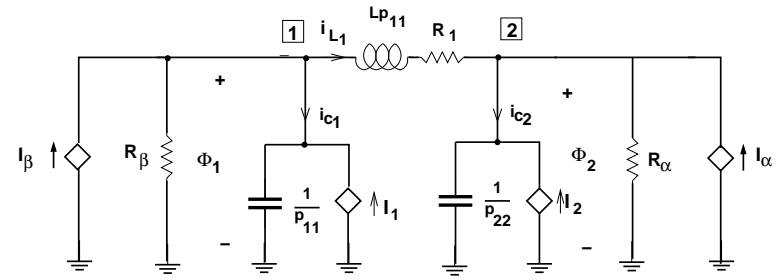

Fig. 2. Left PEEC circuit with models for both transmission condition

\section{TRANSMISSION CONDITIONS FOR WR}

The classical WR approach for partitioning usually consist of breaking a connection between multiple SSys. In our example of the PEEC circuit here, we consider the MNA subcircuit matrices of (6)

$$
M_{1}:=\left[\begin{array}{ccc}
\frac{s}{p_{11}} & 1-\frac{p_{12}}{p_{11}} e^{-s \tau_{12}} & 0 \\
1 & -s L p_{11}-R_{1} & -1 \\
0 & 1-\frac{p_{21}}{p_{22}} e^{-s \tau_{12}} & \frac{s}{p_{22}}
\end{array}\right]
$$

and

$$
M_{2}:=\left[\begin{array}{ccc}
\frac{s}{p_{22}} & 1-\frac{p_{23}}{p_{22}} e^{-s \tau_{23}} & 0 \\
1 & -s L p_{22}-R_{2} & -1 \\
0 & -1+\frac{p_{32}}{p_{33}} e^{-s \tau_{23}} & \frac{s}{p_{33}}
\end{array}\right]
$$

and the coupling vectors

$$
\boldsymbol{m}_{1}:=\left[\begin{array}{c}
\frac{p_{12}}{p_{11}} e^{-s \tau_{12}}-\frac{p_{13}}{p_{11}} e^{-s \tau_{13}} \\
-s L p_{12} e^{-s \tau_{L}} \\
\left.1-\frac{p_{23}}{p_{22}} e^{-s \tau_{23}}\right)^{T}
\end{array}\right]
$$

and

$$
\boldsymbol{m}_{2}:=\left[\begin{array}{c}
1-\frac{p_{21}}{p_{22}} e^{-s \tau_{12}} \\
-s L p_{21} e^{-s \tau_{L}} \\
\frac{p_{31}}{p_{33}} e^{-s \tau_{13}}-\frac{p_{32}}{p_{33}} e^{-s \tau_{23}}
\end{array}\right]
$$

To solve the partitioned problem, the far current at the side of the branch is first set to zero until updated voltages are available from the WR iterations. Denoting the unknowns of the first sub-circuit by $\boldsymbol{x}\left(s_{1}\right):=\left(\Phi_{1}\left(s_{1}\right), i_{L 1}\left(s_{1}\right), \Phi_{2}\left(s_{1}\right)\right)^{T}$, and the unknowns of the second subsystem by $\boldsymbol{x}\left(s_{2}\right):=$ $\left(\Phi_{2}\left(s_{2}\right), i_{L 2}\left(s_{2}\right), \Phi_{3}\left(s_{2}\right)\right)^{T}$, and similarly for $\boldsymbol{b}$, we obtain the classical WR algorithm

$$
\begin{aligned}
& M_{1} \boldsymbol{x}^{k}\left(s_{1}\right)=\boldsymbol{b}\left(s_{1}\right)-\boldsymbol{m}_{1} i_{L 2}^{k}\left(s_{1}\right), \\
& M_{2} \boldsymbol{x}^{k}\left(s_{2}\right)=\boldsymbol{b}\left(s_{2}\right)-\boldsymbol{m}_{2} i_{L 1}^{k}\left(s_{2}\right),
\end{aligned}
$$

where $k$ is the WR iteration index. The unknown currents $i_{L 2}^{k}\left(s_{1}\right)$ and $i_{L 1}^{k}\left(s_{2}\right)$ on the right are relaxed to the previous iteration, by imposing the transmission conditions

$$
\begin{aligned}
& i_{L 2}^{k}\left(s_{1}\right)=i_{L-1}^{k-1}\left(s_{2}\right), \\
& i_{L 1}^{k}\left(s_{2}\right)=i_{L 2}^{k}\left(s_{1}\right) .
\end{aligned}
$$

Fig. 2 represents an equivalent circuit for the left SSy, provided the resistor connection on the right is removed. In the classical case only current or voltage is transmitted across the interface, and the resistor is key for a better method. The oWR approach is based on exchanging more than one variable, for which the impact is optimized. For the problem at hand, we subdivide the system into the same two SSys as before, but for reasons which 
will be apparent below, we now use the following transmission condition between SSy1 (s1) and SSy2 (s2):

$$
\begin{aligned}
i_{L 2}^{k}\left(s_{1}\right)+G_{\alpha} \Phi_{2}^{k}\left(s_{1}\right) & =i_{L 2}^{k-1}\left(s_{2}\right)+G_{\alpha} \Phi_{2}^{k-1}\left(s_{2}\right) \\
i_{L 1}^{k+1}\left(s_{2}\right)+G_{\beta} \Phi_{2}^{k}\left(s_{2}\right) & =i_{L 2}^{k}\left(s_{1}\right)+G_{\beta} \Phi_{2}^{k}\left(s_{1}\right)
\end{aligned}
$$

We show in Fig. 2 the circuit for the left SSy which includes the resistor on the right corresponding to the new transmission conditions. The parameters which are optimized in this new circuit are $G_{\alpha}$ for one direction and $G_{\beta}$ for the coupling in the other direction. It is obvious from this why we call the adjustable parameters conductances.

\section{IMPROVED CONVERGENCE FOR OWR}

In this section we illustrate the improved convergence of the $o W R$ algorithm. The introduction of the conductances $G_{\alpha}$ and $G_{\beta}$ gives us two circuit elements which we can choose in order for the algorithm to converge more rapidly. We illustrate this for the model circuit in Fig. 1 with circuit element values chosen to be

$$
\begin{gathered}
L p_{11}=0.022362, L p_{12}=0.006314 \\
L p_{21}=0.006314, L p_{22}=0.022362 ; \\
p_{11}=1.19143, \quad p_{12}=0.300756, \quad p_{21}=0.300756, \\
p_{22}=0.80392, \quad p_{33}=1.19143, \quad p_{13}=0.121378, \\
p_{31}=0.121378, p_{23}=0.300756, p_{32}=0.300756 ; \\
R_{1}=0.001, R_{2}=0.001 ; \\
t_{12}=0.167, t_{13}=0.333, t_{23}=0.167, t_{L}=0.167
\end{gathered}
$$

Using substantial analysis, which is beyond the scope of this short paper, we can compute for both the classical WR and the oWR the contraction factor $\rho(\omega)$ for each frequency $s=i \omega$, i.e. the number with which the error in that frequency component is multiplied for each iteration of the algorithm. One can show that $\rho(\omega)=\rho(-\omega)$, and since we compute on a bounded time interval $(0, T)$, in our example $T=5$, and the error for $t=0$ vanishes, since the initial condition is known, the frequency range is bounded by a lowest frequency $\omega_{\min }>0$. We show in Fig. 3 a comparison of the contraction factor of the classical WR and the optimized WR, where we optimized the contraction factor using the conductances $G_{\alpha}$ and $G_{\beta}$ minimizing the contraction factor of oWR we know analytically. For the circuit element values we have chosen, the best conductances were found to be $G_{\alpha}=-1.2412$ and $G_{\beta}=8.1169$. One can clearly see that classical WR is not convergent for the critical angular frequencies $\omega$ around \pm 10 , the contraction factor is bigger than one there, and hence the method would diverge for these frequencies, if one computed over a very long time interval. This is different for oWR, which has a substantially improved contraction factor: it is now uniformly less than one, which means all frequencies $\omega$ are convergent. We see that the optimal choice of $G_{\alpha}$ and $G_{\beta}$ leads to equioscillation of the convergence factor: it is the same for the lowest and the difficult frequency around $\omega=10$. We note that on a bounded time interval even classical WR will converge, because on bounded time intervals, WR algorithms converge ultimately superlinearly, and even modes that have a contraction factor bigger than one in our analysis will

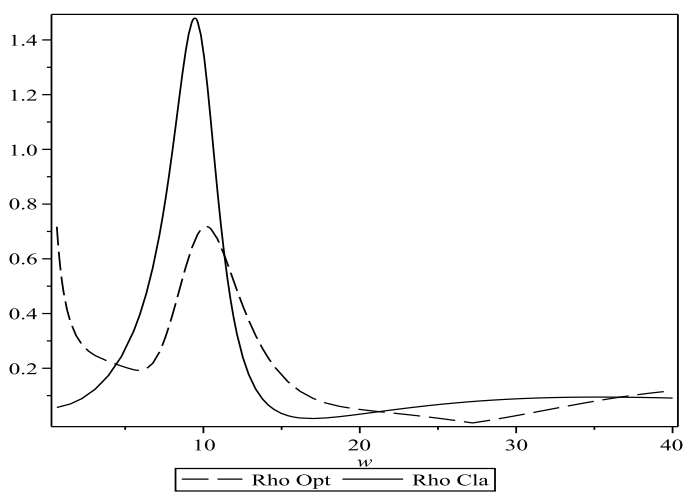

Fig. 3. Comparison of the contraction factor of the classical WR and the oWR algorithm, for each angular frequency $\omega$

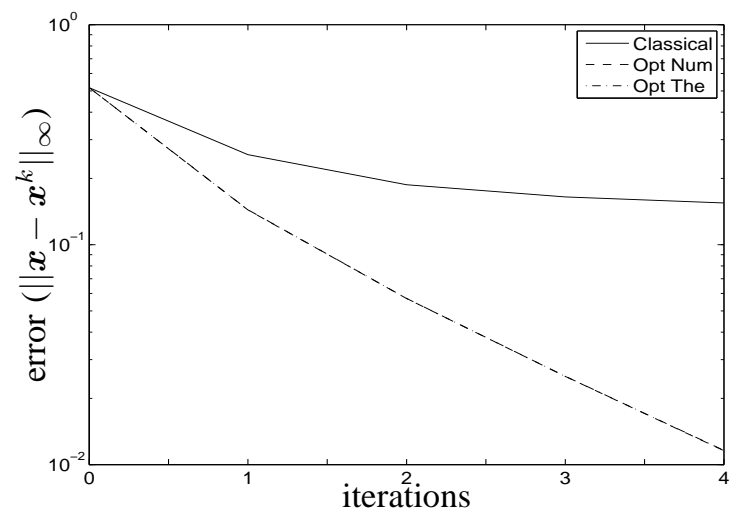

Fig. 4. Convergence behavior of the classical (top curve) and optimized WR algorithm for the theoretically optimized $G_{\alpha}$ and $G_{\beta}$ and their numerically optimized counterparts, which are very close (bottom curves)

eventually converge. We show next a numerical experiment to illustrate that this however slows down convergence.

We discretize the MNA equations for the model circuit in Fig. 1 using a backward Euler discretization with time step $\Delta t=1 / 768$, and we use as the input current $I_{s}$ a linear hat function, which grows for $0<t<0.05$ to 1 , and then decreases for $0.05<t<0.1$ back to zero, where it remains for $t>0.1$. We simulate the circuit on the time interval $(0, T)$ with $T=5$. We show in Fig. 4 the convergence behavior of the classical algorithm compared to the theoretically optimized one with $G_{\alpha}=-1.2412$ and $G_{\beta}=8.1169$, and the best possible choice of the conductances, which was found by running the algorithm and using numerical optimization to minimize the error $\left(\left\|\boldsymbol{x}-\boldsymbol{x}^{k}\right\|_{\infty}\right)$ after 4 iterations, and led to $G_{\alpha}=-1.1723$ and $G_{\beta}=8.359$. We can clearly see that the theoretical optimization found a very good set of parameters, the algorithm can not perform better than with the parameters determined theoretically.

\section{NUMERICAL EXAMPLE RESULTS}

We choose the two conductor problem in Fig. 5 to illustrate the partitioning of a strong $d c$ coupled path. It is important to include the full wave retardation between the elements for the relatively fast rise time of the applied signal. The structure which is $50 \mathrm{~mm}$ long is excited with a linear ramp current 


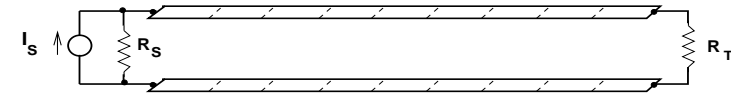

Fig. 5. Example PEEC problem with two wires

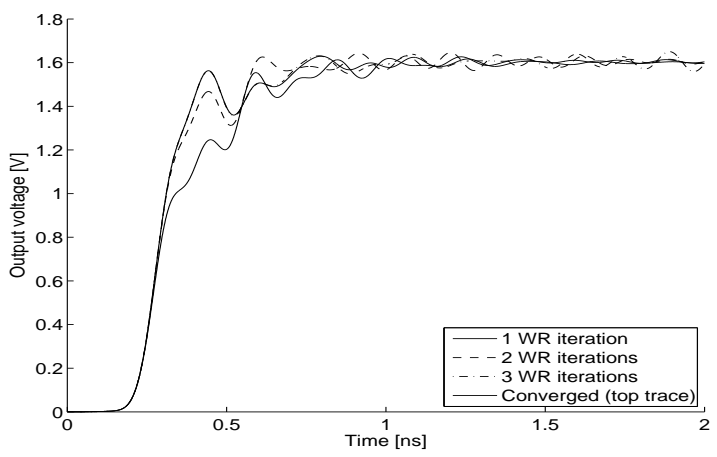

Fig. 6. Convergence with good convergence conductances

source which has a $200 \mathrm{ps}$ rise time. The magnitude of the current is $10 \mathrm{~mA}$. We represent each wire with 10 PEEC cells as shown in Fig. 5, which is relatively few. The flat wires are 2 $\mathrm{mm}$ wide and are spaced $5 \mathrm{~mm}$ and the two wires are connected together with $R_{T}=0.2 \mathrm{kOhms}$. For the experiments, we subdivide the structure into two parts, where each of the parts has 5 sections. All the far coupled partial inductances and capacitive current sources are taken into account by using classical WR as it was done in [14], where it was shown that the far coefficient WR convergence is extremely fast and that we can determine approximate convergence conditions. In Fig. 6 we show that oWR converges to the solution in very few iterations. For this case, the optimized conductances are $G_{\alpha}=-1.9$ and $G_{\beta}=2.4$. As a sensitivity experiment, we increased both values 5 times and the results in Fig. 7 show that, while the convergence is less uniform, it is still quite fast. In Fig. 8, we show that for the case where $G_{\alpha}=0$ and $G_{\beta}=2.4$, the convergence is very slow, as expected. This illustrates the effectiveness of the oWR approach presented in this paper.

\section{CONCLUSIONS}

The oWR method given in this paper represents a major improvement in the convergence rate for waveform relaxation.

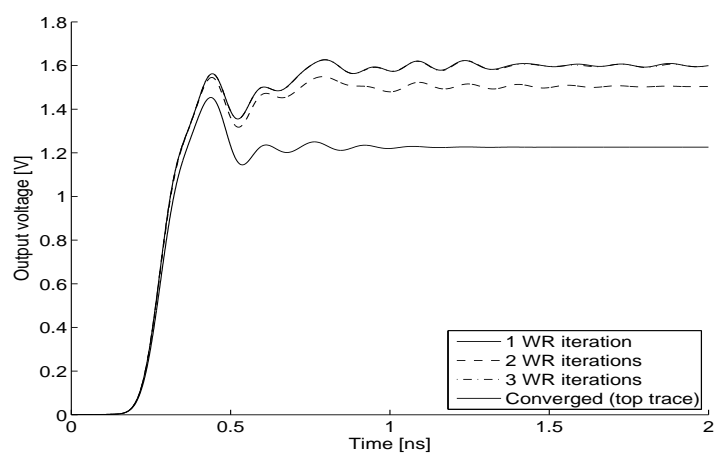

Fig. 7. Convergence with five times larger convergence conductances

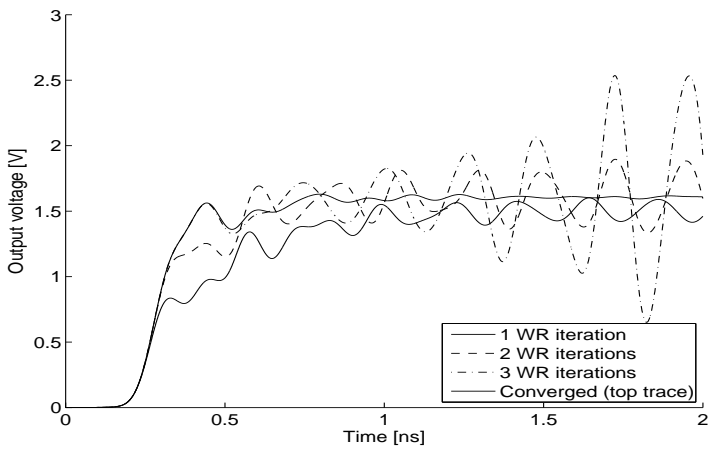

Fig. 8. Convergence test with forward optimization turned off

A new circuit interpretation is given for the transmission conditions. It is also shown that the WR approach can successfully be applied to delayed full wave PEEC models. Further, the models which before did converge very slowly can converge in very few iterations. This is an important step forward since this approach is key for multiprocessor computing.

\section{REFERENCES}

[1] A. E. Ruehli, G. Antonini, J. Esch, J. Ekman, A. Mayo and A. Orlandi. Non-orthogonal PEEC formulation for time and frequency domain EM and circuit modeling. IEEE Transactions on Electromagnetic Compatibility, 45(2):167-176, May 2003.

[2] E. Lelarasmee, A. E. Ruehli, and A. L. Sangiovanni-Vincentelli. The waveform relaxation method for time-domain anaylsis of large-scale integrated circuits. IEEE Trans. on CAD of Integrated Circuits and Systems, CAD-1(3):131-145, July 1982.

[3] H. Peng and C.-K. Cheng. Parallel transistor level fulll-chip circuit simulation. In Design Automation and Test in Europe, pages 304-307, Nice, France, April 2009.

[4] A. E. Ruehli and T. A. Johnson. Circuit Analysis Computing by waveform relaxation, volume 3 . Wiley Encyclopedia of Electrical Electronics Engineering, New York, 1999.

[5] M. J. Gander, L. Halpern, and F. Nataf. Optimal convergence for overlapping and non-overlapping Schwarz waveform relaxation. In C-H. Lai, P. Bjørstad, M. Cross, and O. Widlund, editors, Eleventh international Conference of Domain Decomposition Methods. ddm.org, 1999.

[6] M. J. Gander, L. Halpern, and F. Nataf. Optimal Schwarz waveform relaxation for the one dimensional wave equation. SIAM Journal of Numerical Analysis, 41(5):1643-1681, 2003.

[7] M. J. Gander and L. Halpern. Optimized Schwarz waveform relaxation methods for advection reaction diffusion problems. SIAM J. Numer. Anal., 45(2):666-697, 2007.

[8] M. J. Gander and A. Ruehli. Optimized waveform relaxation methods for RC type circuits. IEEE Transactions on Circuits and Systems, 51(4):755-768, 2004

[9] M. J. Gander M. Al-Khaleel and A. E. Ruehli. Optimized waveform relaxation methods for logitudinal partitioing of transmission lines. IEEE Transactions on Circuits and Systems I, 56(8):1732-1773, 2009.

[10] V.B. Dmitriev-Zdorov and B. Klaassen. An improved relaxation approach for mixed system analysis with several simulation tools. In EURO-DAC '95/EURO-VHDL '95, pages 274-279. IEEE Computer Society Press, 1995.

[11] V. B. Dmitriev-Zdorov. Generalized coupling as a way to improve the convergence in relaxation-based solvers. In EURO-DAC 96/EUROVHDL '96, pages 15-20. IEEE Computer Society Press, 1996.

[12] D. Patterson. The trouble with multi-core microprocessors. IEEE Spectrum Magazine, 47(3):28, July 2010.

[13] N. J. Nakhla, A. E. Ruehli, M. S. Nakhla, R. Achar and C. Chen. Waveform relaxation techniques for simulation of coupled interconnects with frequency-dependent parameters. IEEE Transactions on Advanced Packaging, 30(2):257-269, 2007.

[14] G. Antonini and A. E. Ruehli. Waveform relaxation time domain solver for subsytem arrays. IEEE Transactions on Advanced Packaging, accepted for publication 2010. 